\title{
Survey of facial masks using behaviour during COVID-19 outbreak
}

\author{
Xinkai $\mathrm{Yu}^{1 \#}$, Hao Yang ${ }^{2 \#}$, Junzhou $\mathrm{He}^{3 *}$ \\ ${ }^{1}$ Princeton International School of Mathematics and Science, Princeton, USA \\ ${ }^{2}$ Internatinal Department, The Second High School Attached to Beijing Normal University, Beijing, China \\ ${ }^{3}$ Department of Building Science, Tsinghua University, Beijing, China \\ \#These authors contributed equally to this work. \\ ${ }^{*}$ Corresponding email: hjz16@mails.tsinghua.edu.cn
}

\begin{abstract}
Wearing facial masks has been indicated as an effective way to reduce the risk of COVID-19 transmission among people. To get a deeper understanding of the habit of using face masks in normal life during COVID-19 outbreak and seek ways to solve the problems of mask using, we conducted a large-scale survey in China through online questionnaires. Totally 1004 respondents, including community outreach workers, non-healthcare professionals, retired people, and students participated in this study. Detailed information regarding the types of masks that people usually use, the habit of changing masks, the methods of mask cleaning, and the attitude toward reusing masks and their potential impact on the environment were collected and analyzed. Results show that more than $98 \%$ of people in China used masks during the pandemic, and the types of masks using and frequency of masks changing/reuse varied between different people. Moreover, most of the respondents had a healthy mask-changing habit for preventing the spread of COVID-19 and worried about the safety of mask reusing. The study would help enhance public awareness and develop policies for a better habit in face of the pandemic.
\end{abstract}

\section{Introduction}

The COVID-19 has become a global pandemic since 2020. It cuts off people's face-to-face interactions and largely restricts travel and other communications from different regions. According to the World Health Organization (WHO), there are at least three possible routes of COVID-19 transmission from person to person, including direct or indirect contact transmission, close-range droplet transmission, and airborne transmission 0. Contact transmission is the transmission through direct or indirect contact with the infection sources, such as handshaking with an infector or contact with the contaminated surface or objects. Close-range droplet transmission transfers viruses through large droplets at a short distance, normally happens when a healthy person inhales virus-laden particles from nearby infectors. The airborne transmission could transmit the virus through small droplets or particles emitted by human activities, which can reach a large distance and allows the virus to remain in the air for a long period.

Wearing a facial mask is believed to protect people from being infected thorough restricting all the above three transmission routes. For contact transmission, wearing a facial mask lowers the chance that people may contact their oronasal region, which lowers the infection risks. Wearing facial masks helps protect people from close-range droplet transmission because masks can block the large droplets from reaching the respiratory tract of healthy persons. For airborne transmission, facial masks are also effective in blocking a portion of the small particles. In particular, the N95 facial masks, if correctly used, could remove more than $97 \%$ of small particles 0 . It would be even more important if infectors (unidentified or asymptomatic) were to wear facial masks so that the number of particles released would be reduced. Given the critical importance of facial masks to protect people, it is necessary to better understand people's mask using habits.

This study aims to collect both fundamental information such as mask types, and deeper information about people's mask-using habits such as maskchanging and mask-reuse. The re-use habits of masks are especially worthy of investigating because the service time of a mask is directly related to its effectiveness, and the environmental pollution caused by the abandoned facial masks is also a problem. The investigation was made through online surveys, and the data were collected from late April to early May 2020, in China. Results of this study could help develop better prevention and control guidelines of COVID-19.

\section{Methodology}

We conducted an anonymous electronic questionnaire survey to collect the information. Comparing to the traditional paper questionnaire and face-to-face interviews, the electronic questionnaire has several advantages. First, the feature of anonymous provided respondents privacy so they would not worry 
about their answers being leaked out. Consequently, the respondents would more likely to tell the true information. Moreover, the electronic questionnaire could be easily spread out to more people. Besides, under the circumstance of COVID-19, an electronic questionnaire did not require interaction between people, which eliminated the risk of being infected during the investigation.

The questionnaire contained four types of information: the demographics information, the maskwearing habits, the mask changing frequency and conditions, and the mask cleaning and reuse. The first section provided the basic information of the respondents such as their ages and job types. The information in the second section helped to analyze the fundamental mask-using habits of people. The third and fourth sections allow for understanding the mask change and reuse habits. Multiple types of questions were designed to obtain the information, including filling blank questions, one-choice questions, and multiplechoice questions. The last several questions about the mask reusing habit only appeared when respondents chose to agree with the mask reusing.

The questionnaire surveys were sent out through WeChat, the most popular mobile social application in China. The surveys were made in early 2020, and the questionnaires were designed in Chinese. A total of 1004 responses were collected. The completed questionnaires were automatically stored in a database. The data were then classified and analyzed based on the four sections discussed previously.

\section{Result and Discussion}

\subsection{Demographic Information}

Figure 1 shows a roughly normal distribution pattern of age groups, with 17 to 57 the most represented age range. The results also indicated that the respondents were composed of $73.7 \%$ non-medical working professionals, $5.9 \%$ healthcare professionals, and $20.4 \%$ students. This means that the samples covered people of different ages and professions.

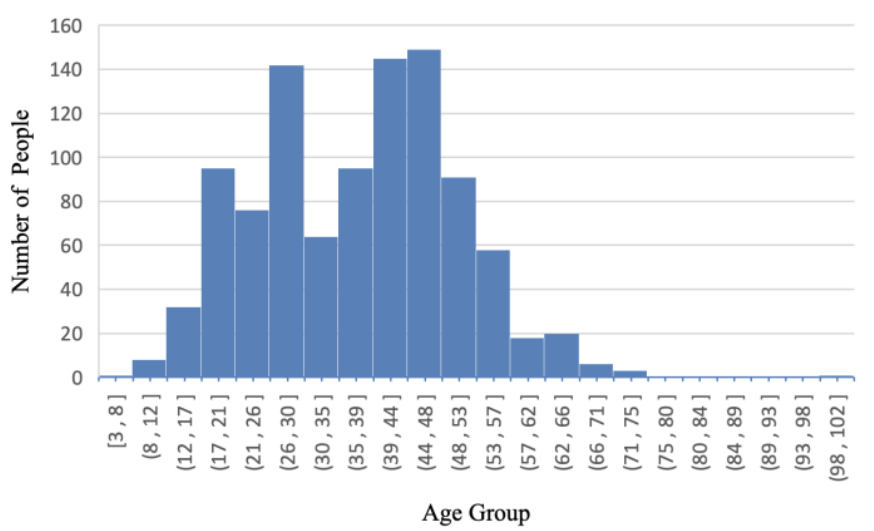

Figure 1. Age distribution of the survey respondents

\subsection{Mask Wearing Habits}

Results show that although most respondents were non-medical professionals, $98 \%$ of them would take on masks when they go outside. The rest $2 \%$ might be due to various reasons such as in shortage of mask supply. Hence, the idea of wearing facing masks when going out was well established in people's minds.

The types of masks used varied from KN95 masks, medical surgery masks, cotton masks, self-made masks, and other types. As shown in Figure 2, 89.5\% used medical surgery masks and $23.0 \%$ of respondents took KN95 masks. The adding of these two numbers already surpassed $100 \%$, because this was a multiple-choice question. Usage of other types was very small compared to the above two types.

Since the investigation was conducted right after the COVID-19 epidemic in China, we speculate that the lack of purchasing access to KN95 led to its low percentage of usage. On the other hand, medical surgery masks were much easier to be purchased. Other types of masks were not favored. The KN95 masks can effectively control aerosol transmission, but they are more costly and uncomfortable when wearing for a long time [2]. Moreover, as the U.S. CDC suggested, the main effect of wearing facial masks is to prevent the spread of droplets from infected person and provide people a psychological hint of social distancing [3]. In this regard, surgical masks could do equally well despite its relatively low filtration efficiency compared to KN95.

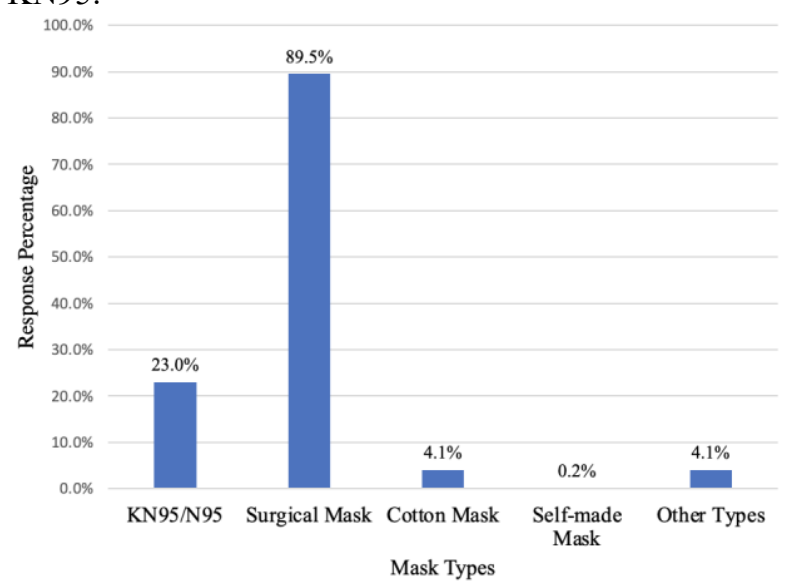

Figure 2. The percentage of usage of different types of masks

\subsection{Mask Changing Frequency and Conditions}

Figure 3 shows that $54 \%$ of the respondents changed masks after using 2-5 times, and $22 \%$ of them changed after wearing only once. For respondents who changed the masks based on using duration, $50 \%$ of them changed after using more than 8 accumulated hours, as Figure 4 shows. The main overlap between the two types of respondents indicated that 2- 5 times of usage would be equivalent to 4-8 hours of accumulated usage or 1-3 days of intermittent usage (not counting the usage hours), which also implied the average using time for each mask wearing. This result also suggested that most people would not throw ways masks after only one time 
use, unless they went to special and "risky" environments such as hospitals. As explained before, the main purpose of masks would be to prevent spread of droplets from potential virus carriers and protect healthy persons from being infected. As most people defined themselves as the latter, it made sense to have multiple time usage unless they justified it as necessary to replace only after short use.

Besides, the result of the question "after going to which place will you change facial mask immediately" displayed in Figure 5 shows that people generally have the awareness of places with high population density or higher chance of infection. Hospitals, public transportation, and places where easily appear gathered people obviously contain the risk factors mentioned above.

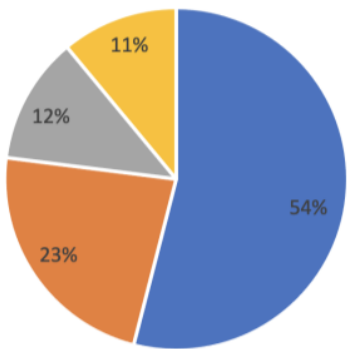

- 2-5 times

- 1 time

- 5-7 times

- More than 7 times

Figure 3. Distribution for habits of mask changing based on using frequency

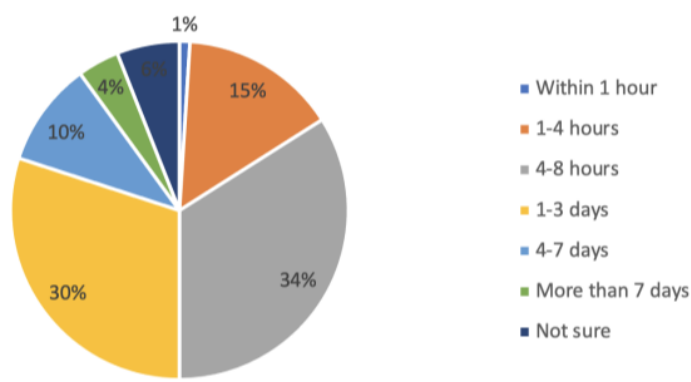

Figure 4. Distribution for habits of mask changing based on length of using time

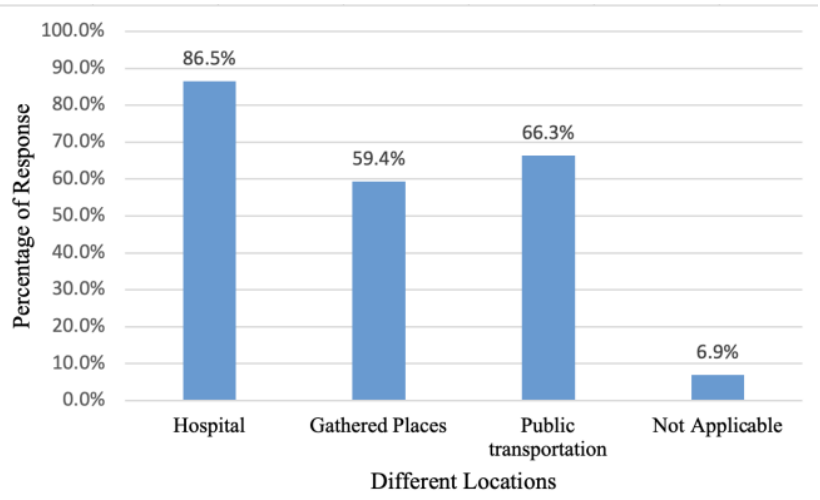

Figure 5. Immediate mask changing after visiting certain places

\subsection{Mask Cleaning and Re-use}

Based on the result of the cleaning and re-use questions, $78.4 \%$ of respondents had concerned about environmental pollution due to abandoned masks. Ionically, only $43.7 \%$ of the respondents agreed with cleaning the masks and re-using them, and merely $21.5 \%$ of respondents did clean masks. This suggests that the idea of mask reuse did not yet reach a general agreement among people. Lack of knowledge and methods about correctly cleaning masks might trigger this phenomenon.

\section{Conclusions}

Based on the results of this on-line survey of mask using habits, it can be concluded that most people understood the importance of wearing masks during the pandemic. The main effect of using masks is to prevent the spread of the droplets from the infected persons and protect healthy people from being infected. The using habits of wearing masks could meet such requirements.

Most of the respondents had a healthy maskchanging habits. For mask types, surgical masks were most favored by people and KN95 masks were the second popular type. Most of the respondents changed masks after using 2-5 times and still, 23\% changed after wearing only once; more than half of them changed after using more than 8 accumulated hours. Besides, the result of places in which people will immediately change masks shows that most people can recognize places with potentially high infection risks, such as hospitals and public transportation.

The investigation also indicated the environmental awareness of people, since $78.4 \%$ of the respondents concerned about the possible environmental pollution brought by large number of abandoned masks. However, only $43.7 \%$ of the respondents agreed with cleaning the masks and re-using them, and merely $21.5 \%$ of respondents cleaned masks. This shows that the idea of mask re-use and cleaning had not yet reached a general agreement among people.

\section{References}

[1] World Health Organization. Transmission of SARS-CoV-2: Implications for Infection Prevention Precautions.

https://www.who.int/newsroom/commentaries/detail/modes-oftransmission-of-virus-causing-COVID-19implications-for-ipc-precautionrecommendations 2021-01-20

[2] Bowen LE. Does that face mask really protect you?. Applied Biosafety 2010, 15(2): 67-71.

[3] Centers for Disease Control and Prevention. Scientific Brief: SARS-CoV-2 and Potential Airborne Transmission https://www.cdc.gov/coronavirus/2019ncov/more/scientific-brief-sars-cov-2.html 2021-01-20 